\title{
Das Lößprofil von Nové Mesto im Waagtal
}

\author{
Von Jiří Kukla: Voj̣en Ložek \& Juraj Bárta \\ Mit 7 Abbildungen, 1 Tafel und 1 Tabelle im Text
}

$\mathrm{Zusammenf}$ assung. - In der Ziegeleigrube von Mnešice bei Nové Mesto nad Váhom (Westslowakei) ist ein reich gegliedertes Lößprofil aufgeschlossen, das für die Stratigraphie der mitteleuropäischen Lößserien von grundsätzlicher Bedeutung ist. Es liegen hier zwei mächtige fossile Bodenkomplexe vor, deren Basisschichten hochinterglaziale Molluskenfaunen mit Helicigona banatica (Rssm.) führen. Im oberen Abschnitt des jüngeren Bodenkomplexes wurde das $\mathrm{S} z \mathrm{el}$ e tien und tiefer das Moustérien festgestellt. Der basale Lessivé und sein unmittelbares Liegendes mit Banatica-Fauna gehören dem letzten Interglazial an, während der obere Abschnitt auch Bodenbildungen des „W 1/2-Interstadials" umfaßt. Ein Frühwürmlöß („W 1 - Löß“) ist hier nicht vorhanden.

Im hangenden Lößpaket ist noch ein schwach ausgebildeter Bodenkomplex vorhanden, der das Gravet ti e n enthält und dem Paudorfer Interstadial entspricht.

Der ältere, ebenfalls sehr mächtige Bodenkomplex ist durch zwei Lessivé-Böden gekennzeichnet. In seinen oberen Schichten wurde das Levallo is ie n, in den unteren das Cla ctonien nlachgewiesen. Unter dem älteren Lessivé treten noch kalkhaltige Schichten mit verstreuten warmzeitlichen Mollusken auf.

Aus dem Vergleich mit klassischen Lößprofi'en Böhmens und Mährens geht hervor, daß der untere große Bodenkomplex von Nové Mesto einer Warmzeit entspricht, die älter als das EemInterglazial, aber jünger als die der Elster-Vereisung unmittelbar nachfolgende Warmzeit ist. Es liegt hier offenbar das Ohe- bzw. Altriß/Jungriß-Interglazial oder ein jüngerer selbständiger Abschnitt der Holstein-Warmzeit vor. Dem Lößprofil von Nové Mesto ist eine Sonderstellung im Raum des Karpatenbeckens beizumessen, da es nicht nur fünf paläolithische Horizonte, sondern auch zwei paläontologisch belegte interglaziale Schichtpakete enthält.

A bstract. - In the brickyard at Mnešice near Nové Mesto on Váh (Western Slovakia) a differentiated loess profile was exposed which is of primary importance for the stratigraphy of loess series in Central Europe. In this profile two thick fossil soil complexes are to be seen, whose basal layers contain molluscan fauna of maximum interglacial with Helicigona banatica (Rssm.).

In the upper part of the younger soil complex $\mathrm{Szelet}$ ian and in the lower layers $\mathrm{M}$ ou $s$ tér i a n were established. The basal lessivé with the underlying beds yielding Banatica-fauna can be placed in the Last Interglacial, whereas the upper section comprises also soils of "W $1 / 2$ Interstadial" age. The early Würmian loess is here not present. In the overlying loess a soil complex corresponding to the Paudorf Interstadial is weakly developed in which $\mathrm{Gr}$ a v e t $\mathrm{t}$ i a $\mathrm{n}$ was found.

The earlier soil complex of great thickness is characterized by two lessivé soils. In the upper

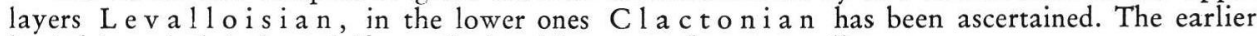
lessivé is underlain by calciferous beds with scattered warm molluscs.

From the comparison with classic loess profiles in Bohemia and Moravia it follows, that the lower thick soil complex in Nové Mesto on Váh corresponds to the Interglacial which precedes the Eemian Interglacial but is younger than the interglacial following immediately the "Elster" glaciation. It is evident that the "Ohe- or Altriss/Jungriss" Interglacial or a younger separate section of the "Holstein" Interglacial is here concerned. The loess profile at Nové Mesto occupies a special position in the area of the Carpathian Basin with regard to the fact that it contains not only five Palaeo!ithic horizons but also two successive interglacial complexes palaeontologically evidenced.

\section{Einleitung}

Die Lößprofile beeiderseits des Waagtales zwischen Trenčín und Hlohovec zogen die Aufmerksamkeit der Forscher in der Zeit zwischen den beiden Weltkriegen auf sich, als hier eine ganze Reihe paläolithischer Rastplätze entdeckt worden war. Mit der Lößstratigraphie befaßte sich hier J. Ретввок $(1935,1936)$, der aber über keine komplizierten Schichtenfolgen berichtet hat. Frste eingehender gegliederte Profile wurden von L. Zотz und W. VLK (1941) aus der Schlucht Hlboký járok bei Moravany und aus Banka beschrieben. Nach dem zweiten Weltkrieg wurden neue Untersuchungen von F. ProšEK und 
V. LožEK durchgeführt, die die Lößaufschlüsse von Banka, Moravany, Zamarovce und Ivanovce untersucht haben (АмBrož, LožEk \& ProšEk, 1952; ProšEk \& LožEk, 1954, 1953). Diese Autoren haben die roten Basisböden, die in Zamarovce durch eine hochinterglaziale Molluskenfauna belegt sind und in Banka paläolithische Artefakte enthalten, in das letzte Interglazial eingeordnet. Im hangenden Schichtpaket wurden fossile Böden des großen Interstadials W $1 / 2$ und des schwachen Interstadials W 2/3 unterschieden. Diese Auffassung übernahm auch J. Pelíšex (1953). P. Woldstedt (1958) führt das Profil von Zamarovce unter den klassischen Profilen dieses Gebietes an.

Im Jahre 1960 beschrieben V. Ložek und J. TYráčEK Profile in der Umgebung von Nové Mesto nad Váhom, und zwar in der Ziegeleei von Mnešice und am Stellwerk Búdy. In der Ziegelei stellten sie an der Basis eines mächtigen fossilen Bodenkomplexes einen zusammenhängenden Horizont mit hochinterglazialer Fauna fest, die in das letzte Interglazial eigestuft wurde. In Anbetracht des Umstandes, daß im Liegenden dieser Interglazialschicht unter einem Lößpaket weitere fossile Böden auftreten (deren Basis während der damaligen Untersuchung noch nicht aufgeschlossen war), bezeichneten sie dieses Profil als stratigraphisch bedeutendsten Aufschluß des untersuchten Gebietes.

Im Laufe der Lehmgewinnung wurden in der Ziegelei von Mnešice auch ältere Schichten aufgeschlossen, so daß es möglich ist, das von LožEK und TYráčEK beschriebene Profil zu ergänzen und genauer zu deuten. Es ist auch gelungen, weitere paläontologische und archäologische Funde zu gewinnen, die für die Lösung der Lößstratigraphie im Waagtal von grundsätzlicher Bedeutung sind. Aus ciner gleichzeitig von J. KukLA durchgeführten Revision anderer Profile ging hervor, daß die stratigraphischen Verhältnisse nicht so einfach sind, wie in älteren Schriften angeführt wurde, und daß die fossilen Böden an der Basis dieser Profile wahrscheinlich um eine Warmzeit älter sind als bisher angenommen wurde.

Aus allen diesen Punkten ergibt sich die außerordentliche Bedeutung des Profils in der Ziegeleigrube von Mnešice. Dort treten zwei mächtige Bodenkomplexe mit paläontologisch belegten Interglazialhorizonten an der Basis auf, in denen die bisher vollkommenste Serie paläolithischer Artefakte verschiedenen Alters gewonnen wurde. Unsere Studie hat sich zum Ziel gesetzt, dieses Stützprofil zu beschreiben, seine Stratigraphie zu lösen und dic Ergebnisse mit anderen Lößprofilen in der Umgebung zu vergleichen. Unser Aufsatz darf zwar nur als vorläufig angesehen werden, allerdings sind die Gesamtverhältnisse in Nové Mesto heute bereits insoweit klar, daß das besprochene Profil als zukünftiger Stratotypus des Waagtalgebietes betrachtet werden kann.

\section{Der Fundort und seine Umgebung}

Das untersuchte Profil befindet sich in der westlichen Ziegeleigrube (Betrieb Nr. 2) am Nordrand des Dorfes Mnešice, einer Vorstadt von Nové Mesto nad Váhom. Mächtige Lösse liegen hier am Fuß der Vorberge der Weißen Karpaten an der Ausmündung des Klanečnica-Tales in das breite Waagtal, das den nördlichsten Ausläufer des Donau-Tieflandes bildet. Die Anhöhe „Na prepadliskách“ $(275 \mathrm{~m})$ nördlich der Ziegelei ist von hellfarbenen Triaskalken aufgebaut, denen z. T. kalkhaltige Konglomerate, Sande und Mergel des Neogens aufliegen. Ein Ausbiß der Kalksteine liegt etwa $100 \mathrm{~m}$ westlich der Lehmgrube.

Außer den Lössen gibt es in der Umgebung keine erwähnenswerten Quartärablagerungen, abgesehen von stellenweise auftretenden Hangbildungen und von den Schottern der Waag-Aue. Im benachbarten Abschnitt des Waagtales liegen weder Terrassen in ursprünglicher Lagerung noch Schotterrelikte bzw. Schotterbestreuung vor, die als Reste der ehemaligen Terrasen betrachtet werden könnten. An beiden Rändern des Waagtales ist aber eine terrassenartige Stufe vorhanden, die zum größten Teil aus Löß besteht. Diese ent- 
stand durch die Seitenerosion der Lößdecke und örtlich auch anderer Bildungen am Rand der Waag-Aue und wurde von M. Lukniš (1946) als „Pseudoterrasse“ bezeichnet.

Das vollständigste Profil liegt an der Nordwand der Grube und kann z. T. auch im benachbarten Abschnitt der Ostwand verfolgt werden. Die Grubensohle liegt durchschnittlich in $205 \mathrm{~m}$ Seehöhe, die Oberkante der Nordwand erreicht 226 bis $229 \mathrm{~m}$. Die Erkundungsarbeiten, die in den Jahren 1957-1958 von L. TKADLEčKOvá (vgl. Geofond FZ 2371) durchgeführt wurden, zeigten, daß die Unterkante der Lößserie etwa in $190 \mathrm{~m}$, also nur wenig höher als die Waag-Aue liegt. Im Liegenden sind im Raum der Ziegelei neogene Tone vorhanden. Daraus ist ersichtlich, daß die Mächtigkeit des Pleistozäns $25-30 \mathrm{~m}$, stellenweise noch mehr beträgt. Die Profile an der Nordwand sind über $20 \mathrm{~m}$ hoch, allerdings stufenartig aufgeschlossen.

Die durchschnittliche Jahrestemperatur ist etwa $9,0^{\circ} \mathrm{C}$, die Niederschläge betragen gegen $700 \mathrm{~mm}$. Die unmittelbare Umgebung der Ziegelei ist entweder verbaut oder landwirtschaftlich bestellt. Wälder xerothermer Prägung mit kleinen Steppenenklaven erhielten sich beiderseits des Waagtales und bedeckten in der Vergangenheit sicher auch den Raum unserer Ziegelei. In höheren Lagen der Weißen Karpaten und des gegenüberliegenden Inovec-Gebirges gehen diese Eichenmischwälder in ausgedehnte karpatische Buchenbestände über.

\section{Beschreibung des Profils}

Die Schichten sind im mittleren Teil der Grubenwand sehr gut aufgeschlossen. Im oberen Arbeitsniveau wird das Material im Winter und im Friihling manuell gewonnen, so daß hier vertikale Wände mit stufenartigen Abständen lange erhalten bleiben. Das gegenseitige Verhältnis aller Schichten war von den älteren zu den jüngsten Serien direkt und ohne Unterbrechung verfolgbar. Obwohl die Abbaufront relativ kurz ist, liegt auch in diesem Abschnitt eine höchst ungleichmäßige Ausbildung der Bodenkomplexe vor. An dieser Stelle scheint eine Erosionsrinne existiert zu haben, die abwechselnd mit Sedimenten aufgefüllt und wieder ausgeräumt wurde, wobei sich ihre Achse mehrmals seitlich verschob. Infolge dieser Verhältnisse blieben in der Lehmgrube außerordentlich gut gegliederte Ablagerungen der ersten Phasen von Zyklus B 1 und C 1 (vgl. J. KukLA, 1961a) in einer Ausbildung erhalten, in der sie bisher nirgends in der Tschechoslowakei bekannt sind. Auch die Mächtigkeit der Bodenkomplexe ist in der Achse der Rinnen außerordentlich groß. Außerhalb der Rinne keilen die Komplexe PK II und PK III (PK = Pedokomplex, Bodenkomplex) in einer Entfernung von nur einigen Metern in eine schwache entkalkte

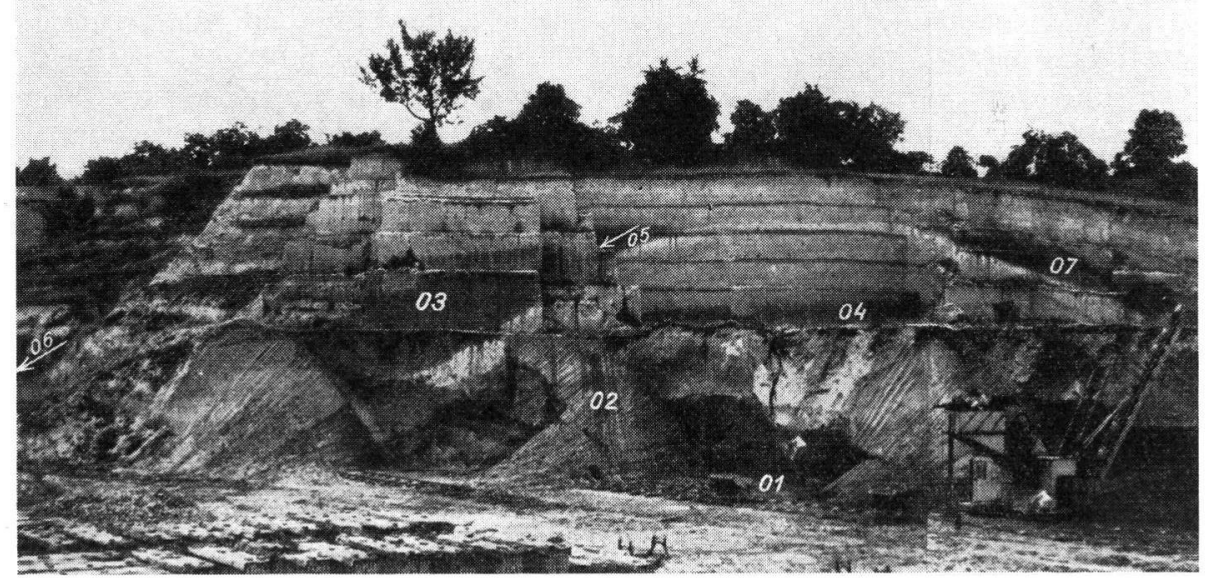

Abb. 1. Nordwand der Ziegeleigrube von Nové Mesto-Mnešice im Sommer 1960 (03,05 - Bezeichnung der Profilabschnitte).

Foto Vojen LožEK. 
Lage aus. Innerhalb der Riß-Lösse verläuft ein Hiatus, dem zeitlich die Entstehung eines tiefen Einschnittes in älteren Ablagerungen einschließlich der Böden von PK IV entspricht. Dieser wurde dann erneut mit Löß und diskordant geneigten Abspülungsbildungen, sowie mit Böden von Zyklus B ausgefüllt.

Tafel 1 gibt das Gesamtprofil der Lehmgrube wieder, das in mehreren Abschnitten aufgenommen wurde; in ihnen weisen die einzelnen Schichtpakete ihre optimale Ausbildung auf. Die Lage dieser Abschnitte ist in der Abb. 1 eingezeichnet. Einzelne Schichten werden in der Tafel kurz gekennzeichnet, so daß hier nur folgende ergänzende Angaben anzuführen sind:

D 3: Die ältesten Lösse D3-a wurden bisher nur in geringer Mächtigkeit von einigen Dezimetern aufgeschlossen. Ihre Oberfläche weist eine starke Kalkanreicherung auf, die den Ca-Horizont der hangenden Böden darstellt. Da es bisher nicht möglich war, petrographische Analysen durchzuführen, kann die Grenze des primären und umgelagerten Lösses nicht genau festgestellt werden. In diesen Übergangsschichten des Lösses D3-a und in den Schwemmschichten der ersten Phase von Zyklus C 1 (C1-a) wurde eine Molluskenfauna (MF 1) mit vereinzelten Interglazialarten gefunden. Da die Mächtigkeit dieser Schichten verhältnismäßig gering ist, ist nicht ausgeschlossen, daß die Fragmente von Interglazialschnecken sekundär durch biologische Tätigkeit in den Fundhorizont verschleppt wurden.

C 1: Ablagerungen der ersten Phase von Zyklus C 1 sind hier bisher im ganzen tschechoslowakischen Gebiet am besten ausgebildet. Auf Abspülungssedimenten liegt ein entkalkter, an der Oberfläche humoser Boden von Pararendsina-Typus (C1-c). In seiner Oberflächenschicht wurden zwei Clacton-Geräte gefunden. Dieser Boden ist von einem feinen homogenen Lehm mit erhöhtem $\mathrm{CaCO}_{3}$-Gehalt überlagert, dessen Habitus primärem Löß entspricht. Bisher wurde aber nicht entschieden, ob hier tatsächlich eine äolische Bildung oder nur ein Schwemmlöß vorliegt. Die folgende Lage (C1-e) besteht aus ausgeprägt feingeschichteten Schwemmlehmen, in welchen Körner von lichtgefärbtem Lehmbröckelsand festgestellt wurden. In diese Schwemmlehme greift die sekundär verlehmte Basis des hangenden Lessivés $\mathrm{C} 1-\mathrm{f}$ ein, dessen Grenze nach unten in unregelmäßigen Keilen einläuft. Es ist interessant, daß die untere Lessivé-Lage einen relativ hohen Humusgehalt aufweist, der offenbar auf durch bodenbildende Vorgänge verursachte Einschlämmung von den Oberflächenschichten her zurückzuführen ist. Im Lessivé gibt es zwei Lagen mit angereichertem Holzkohlenvorkommen, in denen jedoch bisher keine Artefakte gefunden wurden. Die Oberfläche des Lessivé weist kein primäres Gefüge mehr auf und ist wahrscheinlich umgelagert. Sie ist mit kleinen Keilen durchsetzt, die vom Hangenden ausgehen und mit Löß gefüllt sind. Von der Oberkante dieser Schicht bis tief in C1-f reichen weiche, zerfallende Kalkkonkretionen, die entlang der einstigen Wurzelbahnen entwickelt sind. Die jüngste Lage $\mathrm{Cl}$-h besteht an der Basis wahrscheinlich vorwiegend aus Abspülungsmaterial, höher aus hellerem Löß, der Kalkanreicherung aufweist, da er den Karbonathorizont des Lessivés C2-a bildet.

C 2: Im Lessivé C2-a, ebenso wie im liegenden C1-f, sind zerfallende Osteokole erhalten. Seine Oberfläche ist mit feinen, mit Löß bzw. verschwemmtem Lößlehm gefüllten Keilen dicht durchsetzt. Diese Keile sind offenbar ehemalige Trockenrisse und haben also mit einem Frostboden nichts zu tun. Die Übergangszone C2-b besteht aus etwas hellerem, rötlichem Lehm, der ebenfalls mit hellfarbenen Lößkeilchen durchsetzt ist. Es handelt sich wahrscheinlich um eine verschwemmte Lessivé-Oberfläche mit erhöhtem Humusgehalt. Im Profil A wurde in dieser Schicht eine Holzkohlenakkumulation auf einer Fläche von etwa 1 x $1 \mathrm{~m}$ festgestellt, welche örtlich Schmitzen von gebranntem Lehm enthält. In unmittelbarer Nähe dieser vermutlichen Feuerstätte wurden L e v a 11 o is - Geräte gefunden. Die höchste Zone C2-c ist vom Löß gebildet, dessen unterste Lagen durch Absvülung bzw. Bodenfließen gestört sind. 


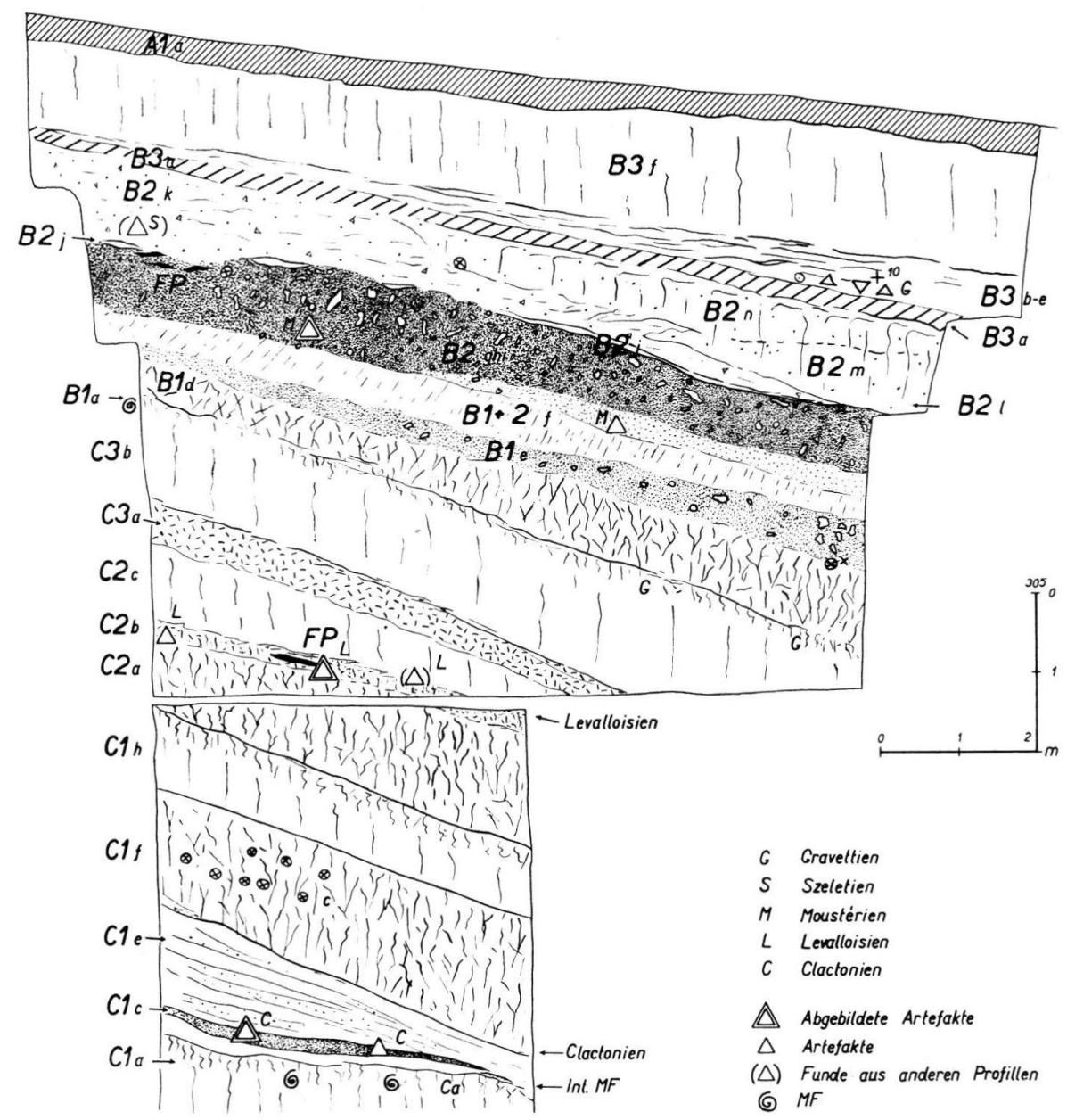

Abb. 2. Profil 07 von Nové Mesto-Mnešice.

C 3: Der Boden C3-a ist nicht vollkommen entkalkt und weist außerdem noch eine sekundäre Kalkanreicherung auf. Sein Karbonathorizont ist deutlich ausgebildet, an der Oberfläche treten Lößkindel auf.

B 1: Die Oberflächenschichten des Lösses C3-b gehen in Lehme mit zahlreichen grünlichen und rostfarbenen Schlieren und Flecken über; eine Schichtung durch Abspülung ist angedeutet (B1-a). Südlich vom untersuchten Profil O3 gibt es Äquivalente dieser Lage mit deutlich ausgeprägter Schichtung. Es handelt sich um basale Sedimente der 1. Phase vom Zyklus B 1, die durch Umlagerung des älteren Lößmaterials entstanden sind. Der obere Abschnitt weist starke sekundäre Kalkanreicherungen auf und enthält eine reiche interglaziale Molluskenfauna (MF 6). Im Profil O5 ist über dieser Zone eine geringmächtige Pararendsina (B1-b) erhalten, die abermals von einem hellfarbenen kalkhaltigen I.ehm überlagert ist (B1-c). Darïber folgt ein Lessivé (B1-d) mit dunkler humoser Oberfläche (B1-e), deren Gefüge aber gleich wie im B-Horizont ist. Diese Zone kann als ein 


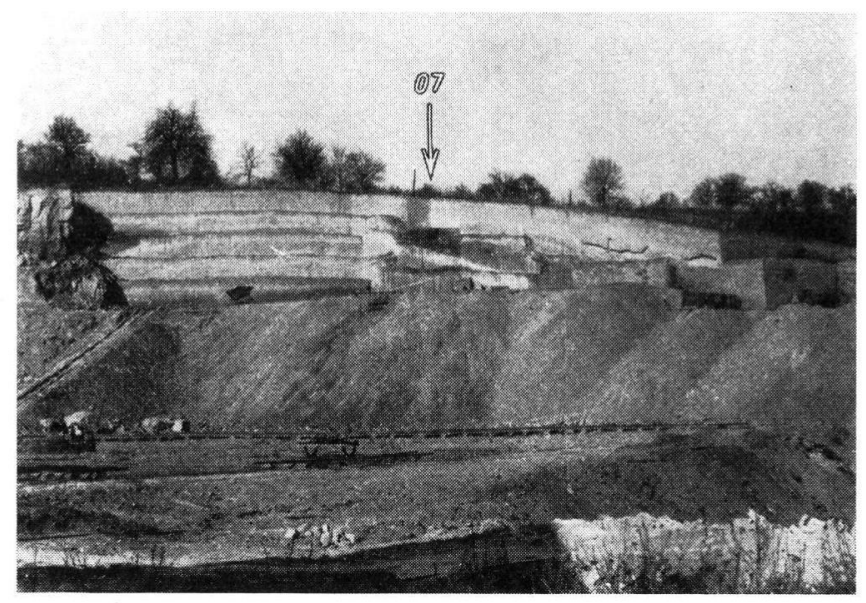

Abb. 3. Ostabschnitt der Nordwand zu Beginn der Rettungsforschung im Januar 1961.

Foto Juraj Bárta.

frühes Entwicklungsstadium von Pseudotschernosem bezeichnet werden. Im Profil O7 ist dieser Horizont als wenig deutlich gefleckter Boden ausgebildet.

B 1 / B 2: Das folgende Schichtpaket ist trotz großer Mächtigkeit schlecht in sich gegliedert. Bisher stehen keine Ergebnisse von petrographischen Untersuchungen zur Verfügung, deshalb mußten wir vorläufig auf eine genaue Bestimmung der Grenze zwischen den Zyklen B 1 und B 2 verzichten. Makroskopisch zeigt das ganze Schichtpaket B 1/2-f Spuren einer schnellen Anschwemmung in der Hanglage, bei der das Material nach Korngröße nicht sortiert wurde. Im gesamten Abschnitt zeigt diese Lage Spuren von Lessivierung. An der Oberfläche (Probe 11) gibt es hellfarbene Filme von ausgewaschenem feinem Mineralstaub, die man an den Absonderungsflächen verfolgen kann. Es fehlt aber ein deutlicher Ae-Horizont des Lessivés. Die Lehme weisen eine grobe senkrechte Absonderung auf, aber die BL.P-Coatings an den Spalten sind nur sehr selten und fein. Wie an Hand der Analysen nachgewiesen werden kann, hat der mittlere Abschnitt dieses Schichtverbandes einen höheren Humusgehalt, eine Erscheinung, die sowohl auf primäre Bodenbildungsvorgänge als auch auf Sedimentation zurïckzuführen ist.

B 2 - B2-g ist eine humose Lage, die unkenntlich in das Liegende übergeht und lokal als spärlich gefleckter Boden ausgebildet ist. Verglichen mit allen älteren Schichten weist dieser Horizont zum erstenmal eine beträchtlichere Humuskonzentration auf. Allerdings ist der Boden in Anbetracht seines Tongehaltes und Kalkmangels als ein Pseudotschernosem zu deuten. Es folgt eine hellere Lehmschicht mit schwach angedeuteten Aggregaten und ein neuer dunkler humoser, ausgeprägt gefleckter Boden (B2-i). Seine Oberfläche ist von typischem Marker (B2-j) und Lehmbröckelsanden überdeckt (B2-k).

Im Schichtabschnitt B 1/2-f bis B2-k können die Lagen B2-j und B2-k ziemlich sicher mit mährischen und böhmischen Profilen parallelisiert werden. B2-g und B2-i entsprechen sehr wahrscheinlich dem humosen Doppelboden von Zyklus B 2 an klassischen Fundstellen Böhmens und Mährens. Fraglich bleibt die Position der verlehmten Zone der 2. Phase von Zyklus B 2 und des Lösses von Zyklus B 1. Die Schichten B 1/2-f könnten zwar nur als verlehmte Basiszone von Zyklus B 2 gedeutet werden, wir sind aber der Meinung, daß hier ein viel komplizierterer Bodenbildungs- und Sedimentationsvorgang vorliegt. Im nahen Aufschluß beim Stellwerk Búdy ist nämlich zwischen dem Lehm, welcher dem unteren Abschnitt von B 1/2-f entspricht und zwischen dem gefleckten Humusboden im Hangenden eine geringmächtige Lößzwischenlage lokal entwickelt. Im Profil O7 fließen 
die Humuszonen B2-g, -h und - $\mathrm{i}$ in einen einzigen, ausgeprägt rostbraun gefleckten Boden zusammen, dessen Liegendes eine deutlich hellere Färbung aufweist. Erst tiefer folgt ein toniger Lehm gleicher Prägung, der dem der Schicht B 1/2-f gleicht. Wir dürfen danach vermuten, daß im Abschnitt O3 die Schicht B 1/2-f nicht nur die 4. und 5. Phase von Zyklus B 1, sondern auch den Löß dieses Zyklus und die verlehmte Basiszone des folgenden Zyklus B 2 einschließt.

Die Oberfläche der Abspülschicht B 2-k ist im Profil O7 stark durch Solifluktion angegriffen. Unter anderem wurde das Abrutschen von flachen, scharf begrenzten Schollen hangabwärts beobachtet. Der hangende Löß B 2-ln ist im Hauptprofil makroskopisch homogen, während im Profil O7 in ihm ein wenig dunklerer humoser gefleckter Boden mit hellfarbenen, wenig deutlichen Flecken erhalten ist (B2-m). Ein ähnlicher, aber stärker ausgebildeter Boden ist in entsprechender stratigraphischer Position von Čermaň bei Nitra bekannt.

B 3: Der Bodenkomplex PK I liegt zwar in unvollkommener, jedoch charakteristischer Ausbildung vor. Der Boden ist nicht ganz entkalkt, besitzt aber einen deutlichen Karbonathorizont, der schlierenartiges Hakenwerfen aufweist. Der hangende Löß ist grau und rostfarben gefleckt und kann mit G 1 B. KI.ímA's (1957) parallelisiert werden (unser B3-de). Besser ist PK I im Profil O7 erhalten. Dort liegt dem gräulich gefärbten parautochthonen Basisboden mit vereinzelten Holzkohlen eine rostfarbene $10 \mathrm{~cm}$ mächtige Zone auf, in der die ältesten Artefakte im Bereich dieses PK gefunden wurden (wahrscheinlich G rave t t e-Kerne). Es folgen Lösse, die basal durch Abspülung feingeschich-

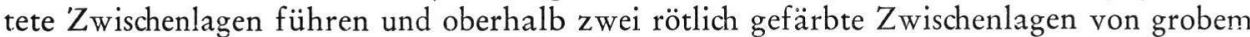
Sand einschließen. Diese sind vermutlich Abspülschichten. Etwa $50 \mathrm{~cm}$ höher liegt im Löß eine rostfarben und grau gefleckte, unscharf begrenzte Zone von initialem Pseudogley, die mit G 2 und G 3 KLímA's (1957) parallelisiert werden kann.

Die Lösse B3-f weisen in der gesamten Mächtigkeit eine gelb-grünliche Tönung auf, die für diese Lößstufe im Waagtal kennzeichnend ist.

A 1: Im Gegensatz zu den älteren Aqquivalenten liegt der holozäne Boden nur ais schwach ausgebildete Parabraunerde vor. Der humose Oberboden ist durch Ackerbau gestört. Altholozäne oder spätglaziale Ablagerungen, also Bildungen der 1 . Phase des Zyklus A 1, wurden bisher im Raum der Ziegelei nicht beobachtet.

\section{Parallelisierung mit klassischen Profilen in Böhmen und Mähren}

Wie im Aufsatz über die Stratigraphie der tschechoslowakischen Lösse (KUKLA, LožEK \& ZÁRUBA, 1961) ausgeführt wurde, haben die einzelnen verschiedenaltrigen Bodenkomplexe im Löß charakteristische Merkmale, anhand welcher sie gegenseitig unterscheidbar und parallelisierbar sind. Das Profil von Nové Mesto ist von diesem Standpunkt sehr günstig ausgebildet.

Am wertvollsten ist unter den Horizonten des jüngeren Abschnittes der Marker, d. h.

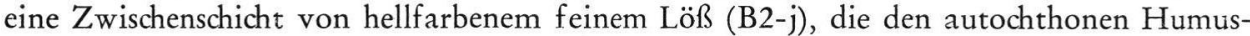
boden von den hangenden Lehmbröckelsanden trennt. In vollkommen entsprechender Position ist er z. B. in Modřice bei Brünn oder in Sedlec und Letky bei Prag erhalten (KukLA, 1961). Er erlaubt uns, beide humosen Böden B2-g und B2-i mit den zwei- bzw. drei interstadialen Tschernosemen in Sedlec bei Prag oder in Unter-Wisternitz, eventuell auch in Leitmeritz I (LožEK \& KukLA, 1959), ziemlich sicher zu parallelisieren.

Auch der basale Lessivé von PK II + III, der hier sogar von hochinterglazialer Molluskenfauna (MF 6) begleitet wird, kann sicher parallelisiert werden. Wir bemerken, daß in entsprechender Lage, d.h. unter der Basis von Böden der Lessivé-Entwicklungsreihe, die Interglazialfauna auch von Leitmeritz II und Letky bei Prag bekannt ist. 
Tabelle der Molluskenfunde

\begin{tabular}{|c|c|c|c|c|c|c|c|c|c|c|c|c|}
\hline \multirow{2}{*}{\multicolumn{2}{|c|}{ Biotop }} & \multirow[b]{2}{*}{ Artenverzeichnis } & \multicolumn{10}{|c|}{ Schichten- und Faunenbezeichnungen } \\
\hline & & & $\begin{array}{l}\text { D3-a } \\
\text { C1-a } \\
\text { MF 1 } \\
\end{array}$ & $\begin{array}{l}\text { C1-e } \\
\text { MF } 2 \\
\end{array}$ & $\begin{array}{l}\text { C1-h } \\
\text { MF } 3 \\
\end{array}$ & $\begin{array}{l}\text { C2-b } \\
\text { MF } 4 \\
\end{array}$ & $\begin{array}{l}\text { C2-c } \\
\text { MF } 5 \\
\end{array}$ & $\begin{array}{c}\text { B1-abc } \\
\text { MF } 6\end{array}$ & $\begin{array}{l}\text { B2-k } \\
\text { MF } 7\end{array}$ & $\begin{array}{r}\text { B2-lmn } \\
\text { MF } 8\end{array}$ & $\begin{array}{c}\text { B3-abcde } \\
\text { MF } 9\end{array}$ & $\begin{array}{l}\text { B3-f } \\
\text { MF } 10\end{array}$ \\
\hline $\mathrm{F}$ & + & Succinea oblonga Draparnaud & 5 & - & 1 & - & - & - & - & - & - & 7 \\
\hline $\mathrm{Sp}$ & $(+)$ & , putris (LINNÉ) & - & - & - & - & (1) & - & (1) & - & - & - \\
\hline M & $(+)$ & Cocblicopa lubrica (MüLLER) & 20 & - & 2 & - & - & - & 6 & - & - & - \\
\hline $\mathrm{X}$ & & lubricella (PorRo) & - & - & - & - & (1) & 3 & - & - & - & - \\
\hline S & $(+)$ & Abida frumentum (DrapaRnaUd) & - & - & - & - & - & 1 & 9 & - & - & - \\
\hline $\begin{array}{l}\mathrm{O} \\
\mathrm{O}\end{array}$ & & $\begin{array}{l}\text { Vertigo pygmaea (DRAPARNAUD) } \\
\text { Truncatellina cylindrica (FÉRUSSAC) }\end{array}$ & $\underline{5}$ & - & 1 & 1 & 8 & 3 & 1 & - & - & - \\
\hline $\mathrm{O}$ & + & $\begin{array}{l}\text { Truncatellina cylindrica (FÉRUSSAC) } \\
\text { Pupilla muscorum (LINNÉ) }\end{array}$ & $\overline{42}$ & $\overline{2}$ & $\overline{61}$ & $-\overline{5}$ & 1 & - & 1 & - & - & - \\
\hline $\mathrm{O}$ & ++ & $\begin{array}{l}\text { Pupilla muscorum (LINNE) } \\
\text { "loessica LožEK }\end{array}$ & 42 & $\underline{2}$ & 61 & 53 & 89 & 2 & $\frac{2}{-}$ & - & 1 & $\overline{174}$ \\
\hline $\mathrm{S}$ & + & sterri (VoITH) & 4 & - & - & - & - & 3 & - & - & - & 46 \\
\hline $\mathrm{S}$ & $(+)$ & $" \quad$ triplicata (STUDER) & 14 & 4 & 21 & 28 & 76 & 5 & 4 & - & 1 & 1 \\
\hline W & $!$ & Orcula doliolum (BRUGUIĚRE) & - & - & 1 & - & - & 59 & - & - & - & - \\
\hline Wp & $(+)$ & dolium (DraparnaUd) & 59 & - & 1 & 3 & 27 & 1 & 19 & 1 & - & - \\
\hline $\mathrm{W}^{2}$ & $! !$ & Pagodulina pagodula (DESMOULINs) & - & - & - & - & - & 41 & - & - & - & - \\
\hline $\mathrm{O}$ & ++ & Vallonia tenuilabris (A. BRAUN) & - & - & - & - & - & - & - & - & 1 & 31 \\
\hline $\mathrm{O}(\mathrm{W})$ & $(+)$ &,$\quad$ costata (MüLLER) & 50 & 1 & 7 & 15 & 24 & 19 & 12 & - & 1 & - \\
\hline $\mathrm{O}$ & & $" \quad$ pulchella (MÜLLER) & - & - & 3 & - & 6 & 3 & - & - & - & - \\
\hline W & $!$ & Acantbinula aculeata (MüLLER) & - & - & - & - & - & 6 & - & - & - & - \\
\hline W & $!$ & Ena montana (DraPARNAUD) & - & - & - & - & - & 1 & - & - & - & - \\
\hline W & $!$ & ” obscura (MüLLER) & - & - & - & - & - & 1 & - & - & - & - \\
\hline$S$ & $(+)$ & Chondrula tridens (MÜLLER) & 55 & 10 & 9 & 22 & 44 & 3 & 96 & - & - & - \\
\hline W & $! !$ & Mastus cf. bielzi (KIMAKOWICZ) & - & - & - & - & - & 16 & - & - & - & - \\
\hline W & $!$ & Cocblodina laminata (MONTAGU) & 3 & - & 1 & - & - & 34 & - & - & -_ & - \\
\hline Wp & $(+)$ & Clausilia dubia Draparnaud & 13 & - & - & 11 & 80 & 19 & - & - & - & 2 \\
\hline $\mathrm{W}^{\mathrm{T}}$ & & , cruciata triplicata A. SСHмIDT & - & - & - & - & - & 2 & - & - & - & - \\
\hline $\mathrm{W}(\mathrm{Sp})$ & & ” pumila C. PFEIFFER & - & (1) & (5) & (3) & - & - & 17 & - & - & - \\
\hline W & $!$ & Iphigena latestriata (A. SсHмIDT) & - & - & - & - & - & 1 & - & - & - & - \\
\hline Wp & $!$ & Laciniaria plicata (DRAPARNAUD) & - & - & - & - & - & 32 & - & - & - & $\ldots$ \\
\hline $\mathrm{W}$ & $!$ & $" \quad$ cana (HELD) & - & - & - & - & - & 1 & - & - & - & - \\
\hline W & !! & $" \quad$ stabilis (L. PFEIFFER) & - & - & - & - & - & 10 & 3 & - & -_ & - \\
\hline $\mathrm{W}(\mathrm{Sp})$ & $(+)$ & " turgida (ROSSMÄSSLER) & 1 & - & - & - & - & - & - & - & - & - \\
\hline & $!$ & Ruthenica filograna (ROSSMÄSSLER) & - & - & - & - & 1 & 40 & 11 & - & - & - \\
\hline $\mathrm{M}$ & $(+)$ & Punctum pygmaeum (DrAPARNAUD) & - & - & - & - & - & 1 & - & - & - & - \\
\hline $\mathrm{W}(\mathrm{M})$ & $!$ & Discus rotundatus (MüLLER) & - & - & - & - & - & 15 & - & - & - & - \\
\hline W & !! & ” perspectivus (MüHLFELDT) & - & - & - & - & - & 22 & - & - & - & - \\
\hline
\end{tabular}




\begin{tabular}{|c|c|c|c|c|c|c|c|c|c|c|c|c|}
\hline M & $(+)$ & Perpolita radiatula (ALDER) & 3 & - & - & 2 & 1 & - & - & - & - & - \\
\hline W & $!$ & Aegopinella pura (Alder) & - & - & - & - & - & 6 & - & - & 一 & - \\
\hline $\mathrm{W}(\mathrm{S})$ & $!$ & minor (STABILE) & 3 & - & - & - & - & 8 & - & - & - & - \\
\hline W & $!$ & "nitens (MICHaUd) & 一 & - & - & - & - & (7) & - & - & - & - \\
\hline W & $!$ & "nitens-minor" & - & - & - & - & - & 23 & - & - & - & - \\
\hline W & $!$ & Vitrea diaphana (STUDER) & - & - & - & - & - & 13 & - & - & - & - \\
\hline W & $!$ & "subrimata (REINHARDT) & - & - & - & - & - & 17 & - & - & - & - \\
\hline M & & $» \quad$ contracta (WESTERLUND) & - & - & - & - & - & 44 & - & - & - & - \\
\hline $\mathrm{W}(\mathrm{F})$ & $(+)$ & crystallina (MüLLER) & 9 & - & - & - & - & 33 & 3 & - & - & - \\
\hline W & $!$ & Daudebardia rufa (DRAPARNAUD) & - & - & - & - & - & 37 & - & - & - & 一 \\
\hline & & Limacidae & 14 & 1 & 5 & 18 & 29 & 62 & 10 & - & - & 1 \\
\hline $\mathrm{X}$ & $!$ & Milax cf. rusticus (Millet) & - & - & - & - & - & 2 & - & - & - & - \\
\hline $\mathrm{W}(\mathrm{S})$ & $(+)$ & Fruticicola fruticum (MüLLER) & $(2)$ & - & - & 3 & - & 7 & 3 & - & 一 & - \\
\hline $\mathrm{S}$ & + & Helicella striata (MüLLER) & 3 & 1 & 12 & 13 & 42 & 17 & 3 & - & 2 & - \\
\hline W & $!$ & Trichia unidentata (DRAPARNAUD) & - & - & - & - & - & 5 & 4 & - & 一 & - \\
\hline M & & lubomirskii (SLOSARSKI) & - & - & - & - & 1 & - & - & - & - & - \\
\hline M & + & bispida (LINNÉ) & 28 & - & 4 & (2) & - & - & - & - & 1 & 4 \\
\hline W & $!$ & Monachoides incarnata (MüLLER) & - & - & - & - & - & 38 & - & - & - & 一 \\
\hline $\mathrm{W}(\mathrm{Sp})$ & & vicina (RosSMÄSSLER) & 1 & - & - & - & - & 4 & 6 & - & 一 & 一 \\
\hline WSp & $(+)$ & Perforatella bidentata (GMELIN) & 38 & - & - & - & 6 & 1 & 6 & - & 一 & - \\
\hline WS & & Enomphalia strigella (DraPARNAUD) & 2 & (1) & 1 & 7 & - & 6 & 12 & - & - & - \\
\hline W & $!$ & Helicodonta obvoluta (MüLLER) & - & - & - & - & - & 146 & - & - & - & - \\
\hline W & !! & Soosia diodonta (FÉRUSSAC) & - & - & - & - & - & 17 & - & - & - & - \\
\hline W & !! & Helicigona banatica (RoSSMÄSSLER) & 1 & - & - & - & - & 10 & - & - & - & - \\
\hline $\mathrm{W}(\mathrm{M})$ & $(+)$ & Arianta arbustorum (LINNÉ) & 66 & 1 & (1) & 4 & 1 & (1) & 3 & - & - & - \\
\hline W & $!$ & Isognomostoma personatum (LAMARCK) & - & - & - & - & - & 3 & - & - & - & - \\
\hline $\mathrm{S}(\mathrm{W})$ & !! & Cepaea vindobonensis (FÉRUSSAC) & - & - & - & - & 一 & 10 & - & 一 & 一 & 一 \\
\hline $\mathrm{W}(\mathrm{S})$ & $!$ & Helix pomatia LinnÉ & 一 & - & - & - & - & 5 & 一 & - & - & 一 \\
\hline W & $!$ & Acicula polita (HaRTMANN) & - & - & - & - & - & 18 & - & - & - & - \\
\hline
\end{tabular}

Erläuterungen :

Bezeichnung der Biotope:

$\mathrm{W}=\mathrm{Wald}, \mathrm{WS}=$ Waldsteppe, xerothermer Wald, W(S) $=$ Waldart, hie und $\mathrm{da}$ in die Waldsteppe vordringend, Wp $=$ Wald und Felsen verschiedener Art (auch oberhalb der Waldgrenze), WSp $=$ Auewald, W(Sp) $=$ sehr feuchte Waldstandorte.

$\mathrm{S}=$ Steppe, $\mathrm{O}=$ Steppen und offene Flächen von trockenen Felsen bis feuchten Wiesen.

$\mathrm{Sp}=$ Sümpfe, feuchte Wiesen, Auen, Ufer.

Arten, die sowohl in Wäldern als auch in offenem Gelände leben: $\mathrm{M}=$ mesophile, $\mathrm{X}=$ trockenheitsliebende, $\mathrm{F}=\mathrm{f}$ euchtigkeitsliebende.

(Anm.: Ein Zeichen in Klammer, z. B. W(M), bedeutet, daß die betreffende Art gelegentlich auch den in Klammer angegebenen Standort bewohnen kann).

!! = Leitart der gipfelnden warmfeuchten Perioden (Hochinterglaziale), ! = für feuchte Perioden bezeichnende Art, $++=$ ausgestorbene Lößart, $+=$ bezeichnende Lößart, $(+)=$ Arten, die lokal auch im Verhand der Lößfaunen erscheinen können.

Die Zahlen in Klammer bedeuten eine nur wahrscheinliche Bestimmung. 
Die Einstufung der Böden von Nové Mesto in die Bodenkomplexe I, II und III wird durch folgende übereinstimmende Merkmale belegt:

1) Gut entwickelter Lessivé an der Basis

2) Ausgeprägte autochthone Humusböden in der Mitte

3) Mächtige humose Lehmbröckelsande über den autochthonen Böden.

Eine Abweichung im besprochenen Profil stellt die ungegliederte Schicht B 1/2-f dar, welche ebenfalls Spuren von schwacher Lessivierung aufweist. Eine Analogie dieser Lage ist von Bulhary unweit von Unter-Wisternitz bekannt. Die geringe BLP-Bewegung, die bisher in entsprechenden Zonen von klassischen Fundstellen nicht beobachtet wurde, kann auf die extrem günstige Lage des Profils am Südhang in einer relativ warmen und hauptsächlich feuchten Klimazone zurückgeführt werden.

Auch die Übereinstimmung des älteren PK IV mit PK IV an klassischen Fundorten Sedlec und Letky bei Prag, sowie Červený kopec bei Brünn ist in den Hauptzügen belegt. Dicht übereinander treten hier zwei Lessivés auf, die von Löß und einem schwachen, zum Teil oder vollkommen entkalkten, rostfarbenen Boden überlagert werden. Die Lehmbröckelsande sind sehr schwach ausgebildet oder fehlen. Bemerkenswert ist in Nové Mesto die schwache Ausbildung von humosen Böden über den Lessivé-Horizonten. Diese Erscheinung hat vermutlich allgemeine Gültigkeit für das Waagtal und wird offenbar durch lokale paläoklimatische Faktoren verursacht.

\section{Molluskenfunde}

In mehreren Schichten des Profils wurde eine reiche Schneckenfauna festgestellt. Die Ergebnisse der malakozoologischen Untersuchung sind in der Tabelle der Molluskenfunde (Seite 80/81) zusammenfassend dargestellt, in der auch alle analytischen, namentlich paläoökologischen Angaben angeführt werden. Die Bruchstücke wurden auf ganze Individuen wie in früheren Arbeiten umgerechnet (vgl. z. B. LožEK, TYRÁčEK \& FEjFAR, 1959, S. 185). Hier können wir uns deshalb nur auf eine kurze biostratigraphische Auswertung der Molluskenfunde beschränken.

Von höchster Bedeutung ist MF 6, die an der Basis des oberen Pakets von fossilen Böden festgestellt wurde. Es handelt sich um eine typisch ausgeprägte hochinterglaziale Gesellschaft, was nicht nur durch die Anwesenheit interglazialer Leitarten [Pagodulina pagodula (DEsm.), Mastus cf. bielzi (KIM.), Laciniaria stabilis (L. PFr.), Discus perspectivus (MüHL.), Soosia diodonta (FÉr.) und Helicigona banatica (RSSM.)], sondern auch durch den großen Artenreichtum (insgesamt 52 Arten!) belegt ist. Durch diese hohe Artenzahl unterscheidet sich die besprochene Thanatozönose auffallend von allen übrigen Faunen. Sie stellt eine Waldgesellschaft dar, die einen frischen Laubmischwald bewohnte. Verglichen mit der heutigen Fauna in der Umgebung zeugt MF 6 für ein wärmeres und beträchtlich feuchteres Klima und belegt sicher ein Hochinterglazial. Dieses bietet uns einen Anhaltspunkt für die stratigraphische Lösung des gesamten Profils.

Von Bedeutung ist weiter die Gesellschaft MF 7 in den Lehmbröckelsanden desselben Schichtpakets von fossilen Böden. Es handelt sich um eine Fauna mit geringen Wärmeansprüchen, an welcher sowohl Steppen- [Chondrula tridens (MüLL.), Abida frumentum (Drap.)] und Waldsteppenarten [Euomphalia strigella (Drap.)] als auch mehrere feuchtigkeitsliebende Mollusken, wie Clausilia pumila C. PFr., Monachoides vicina (Rssm.) und Perforatella bidentata (GMELIN), beteiligt sind. Bemerkenswert ist eine schwächere Beimengung von einigen Interglazialarten, wie Ruthenica filograna (Rossm.), Laciniaria stabilis (L. PFr.) und Trichia unidentata (Drap.). Aus dem zahlenmäßigen Anteil geht aber auf jeden Fall das Vorherrschen von bezeichnenden Arten der sog. Tridens- und 
Fruticum-Faunen klar hervor, die für die Übergangsabschnitte zwischen den Warm- und Kaltzeiten bzw. für die "großen“ Interstadiale kennzeichnend sind (LožEK \& KuKLA, 1959). Die Mischgesellschaft von Steppen- und feuchtigkeitsliebenden Schnecken mit beigemengten Interglazialarten kann durch den Umstand erklärt werden ,daß hier Abspülschichten vorliegen, die aus Abtragsmaterial älterer Böden und Ablagerungen einschließlich deren Fossilgehaltes bestehen.

Die Faunen des oberen Lößpakets (MF 8, 9, 10) sind artenarme Gesellschaften einer waldlosen Landschaft, die für kaltes Klima zeugen. Am besten ist MF 10 mit hohem Anteil von Pupilla loessica LožEK und Vallonia tenuilabris (A. BR.) ausgebildet, die eine typische Lößgesellschaft von ausklingendem Hochglazial darstellt. Vollentwickelte ColumellaFaunen wurden bisher an der Fundstelle nicht nachgewiesen. Dies kann offensichtlich auf lokale Verhältnisse zurückgeführt werden.

Aus dem Lößpaket zwischen dem oberen und unteren Bodenkomplex stammt MF 5. Es handelt sich um eine typische Striata-Fauna der mäßig kalten Lößsteppe, welche erkennen läßt, daß der Löß zeitlich nicht in den Hochstand einer Kaltzeit, sondern in eine Randphase fällt, als die Temperaturverhältnisse bereits günstiger waren. Es ist wahrscheinlich, daß manche Arten aus älteren Schichten umgelagert wurden [Rutbenica filograna (Rssm.), Trichia lubomirskii (SLós.) und Truncatellina cylindrica (FÉR.)].

Sehr bemerkenswert sind die Faunen aus dem unteren Bodenkomplex (MF 2, 3), bzw. von dessen Oberfläche (MF 4). Es handelt sich durchweg um Gesellschaften, die einer mäßig kalten Steppe bis Lößsteppe entsprechen und die ähnlich wie MF 7 gedeutet werden können. Man muß die Beimengung von wärmebedürftigen Arten, z. B. Orcula doliolum (BRug.) und Cochlodina laminata (MTG.) in MF 3, die Anwesenheit der Waldsteppenschnecke Euomphalia strigella (Drap.) und das Fehlen von ausgesprochen kälteliebenden Arten hervorheben. Es handelt sich durchweg um Faunen der Randphasen von Warmzeiten, bzw. der frïhen Phase einer Kaltzeit.

Von außerordentlicher Bedeutung ist MF 1 aus lößartigen Schichten in unmittelbarem Liegendem von PK IV. Auch diese Gesellschaft ist von ähnlicher Prägung wie die eben besprochenen Thanatozönosen (MF 2-4), aber der Anteil an feuchtigkeitsliebenden Arten, vor allem Perforatella bidentata (GMEL.) ist erheblich höher und es gibt hier auch Interglazialarten wie Helicigona banatica (Rssm.), Cocblodina laminata (MTG.) und Aegopinella minor (SтAв.). Diese lassen erkennen, daß die Schicht dem Randabschnitt einer großen Warmzeit angehört. Aus der eingehenden vergleichenden Analyse dieser Thanatozönose ergibt sich, daß hier wahrscheinlich die Anfangsperiode eines großen Interglazials vorliegt, die mit der präborealen Phase des Postglazials verglichen werden kann (vgl. LožEK, TYRÁčEK \& FEJFAR, 1959). Obwohl im Bereich von PK IV bisher keine ausgeprägt hochinterglaziale Molluskenfauna festgestellt wurde, kann MF 1 doch als Beleg eines Interglazials gedeutet werden. Die Bedeutung dieser Feststellung ist ganz außerordentlich, wenn wir uns des Umstandes bewußt werden, daß die besprochene Lage im Liegenden eines anderen, sicher belegten Interglazials auftritt.

Auf Grund der eingehenden Molluskenanalyse ist es gelungen, im Profil zwei interglaziale Horizonte zu erfassen, die in beiden Fällen an der Basis von fossilen Bodenkomplexen liegen. Diese Feststellung entspricht vollkommen den Befunden von anderen Orten. Die Hauptphase der Kaltzeit zwischen beiden Interglazialen ist paläontologisch nicht faßbar, da ihre Schichten im Profil offenbar nicht ausgebildet sind. Auch die jüngste Hochkaltzeit im Hangenden des oberen Interglazials liegt nicht in voller Entfaltung vor. Trotz dieser unvollkommenen Ausbildung der kaltzeitlichen Schichtpakete ist im Profil der Wechsel von Interglazialen und Glazialen malakozoologisch sehr anschaulich belegt, und zwar in einer solchen Ausprägung, daß das Profil unter die klassischen Fundstellen Mitteleuropas eingeordnet werden muß. 


\section{Archäologische Funde}

Das reich gegliederte Profil der Ziegelei Nr. 2 von Nové Mesto-Mnešice hat auch die Aufmerksamkeit der Archäologen auf sich gezogen. Bereits im Sommer 1957 während einer gemeinsamen Exkursion mit J. Kozlowski fand J. BÁrta auf der Sohle der untersuchten Lehmgrube einen kernartigen Abschlag aus grauem Radiolarit und auch ein fossiles Tierbein, das nach O. Fejfar einer Equus-Art angehört. Die erwähnten Funde zeugten für die Entdeckung einer neuen paläolithischen Siedlung im Waagtal. Inzwischen zeigte sich aber, daß bereits im Jahre 1944 der Geograph M. LuknIš Lagen von gebranntem Lehm, die vermutlich den PK III + II zuzuordnen sind, entdeckt hat. Vom archäologischen Standpunkt aus betrachtet kann diese Erscheinung als paläolithische Feuerstätte gedeutet werden. Neue Untersuchungen, namentlich im Jahre 1960, brachten weitere Funde

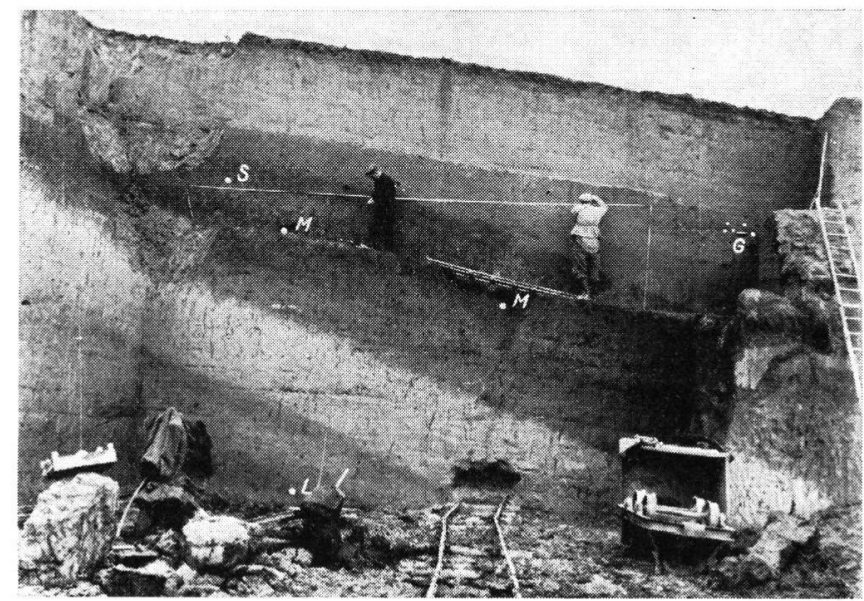

Abb. 4. Profil 07 mit eingezeichneten Artefaktfunden $(G=$ Gravettien, $S=$ Szeletien, $M=$ Moustérien, $\mathrm{L}=$ Levalloisien).

Foto Juraj Bárta.

von Silexen jungpaläolithischer Prägung aus sekundärer Lage, aber auch erste stratigraphisch erfaßte, wenig bezeichnende Klingenabschläge aus dem unvollkommenen Westprofil O6. Diese Funde stammen aus dem oberen Abschnitt des unteren Bodenkomplexes, welcher der Schicht C2-b im Hauptprofil O7 entspricht. Größere Bedeutung gewinnt unsere Fundstelle vor allem aber durch den Artefaktfund aus dem älteren interglazialen Boden von PK IV (Schicht C1-c) etwa 2 m über dem tiefsten Punkt der Grubensohle, der von den Arbeitern der Ziegelei Ende Juli 1960 geborgen wurde. Es handelt sich um einen auffallend breiten, flachen Klingenabschlag ( $\mathrm{Abb}$. 7/4) mit beidseitiger gekerbter, nichtretuschierter Schneide und stumpfem, gröberem und mäßig schrägem terminalem Ende. Dieser Abschlag hat dank der Beschaffenheit des Rohstoffes eine breite, nicht glatte Schlagfläche, welche nur von der Hälfte der Abschlagbreite einen stumpfen Winkel von $118^{\circ}$ mit der Spaltfläche bildet. Ein stark hervortretender Bulbus und mehrere große Schlagnarben, sowie ein Schlagsprung zeugen für das Abschlagen gegen Stein, was außer dem stumpfen Schlagwinkel für das Cla ct o $\mathrm{n}$ bezeichnend ist (Z̈erera, 1952). Das beschriebene Artefakt besteht aus feinkörnigem gragrünem weißkarpatischem Radiolarit (L. 132, B. 78, D. 23), und sein terminales Ende mit weiterem stumpfen Winkel von $100^{\circ}$ weist auf die Möglichkeit einer bipolaren Bearbeitung hin. Das Fehlen der ursprünglichen Oberfläche dieses Rohstoffes ist in diesem Fall darauf zurückzuführen, daß das Artefakt noch vor der Bearbeitung seiner Geröllverwitterungsrınde entkleidet wurde. Als Beweis dieser Voraussetzung darf auch der spätere Fund von zwei großen Radiolaritgeröllen in derselben stratigraphischen Position angesehen werden, wobei eines als grobes Kernstück betrachtet 




Abb. 5. Oberer Abschnitt des fossilen Bodenpakets PK III+II mit dem gefleckten Boden und der Fundstelle des Moustier-Geröllsegmentabschlages.

Foto Juraj Bárta.

werden kann. Dieses zeugt für die Herstellung der C la c t on - Geräte an Ort und Stelle. Ein weiterer Beweis für die Anwesenheit von $\mathrm{Cla}$ ct on an der besprochenen Fundstelle ist der spätere Fund eines Arbeiters der Ziegelei, der in sekundärer Lage unter dem erwähnten interglazialen Boden einen gröberen, aber im ganzen relativ kleinen klingenartigen Abschlag mit feiner partieller sinistrolateraler Retusche und mit allmählich zugespitztem terminalem Ende gefunden hat. Dieser Abschlag aus gelbbraunem Radiolarit hat einen ausgeprägten Schlagkegel und eine deutlich schräge Schlagfläche aus der ursprünglichen Oberfläche des Geröllstückes, wobei diese Fläche mit der Spaltfläche einen stumpfen Winkel von $122^{\circ}$ bildet. Bezüglich seiner Größe (L. 44, B. 27, D. 13) und Form hat dieses Artefakt seine nächste slowakische Analogie im Clacton-Fund von Seňa (Prošex, 1953). Bei der Rettungsforschung des Archäologischen Institutes der SAV in Nitra im Winter 1961 wurden im Hauptprofil O7 im älteren Bodenkomplex (PK IV) in der Schicht C2-b in der Nähe der Feuerstätte zwei ausgeprägte L e va 11 o is - Artefakte zefunden. Ihr Fund belegte stratigraphisch den früheren Fund aus der sekundären Lage, der auch eine ausgesprochene L e v a 11 o is - Bearbeitungstechnik auf wies. Diese stratigraphisch crfaßten Artefakte, namentlich ein breiterer, bzw. längerer klingenartiger Abschlag (Abb. 6c) aus rotbraunem Radiolarit sind durch gleichmäßig gewölbten Bulbus mit Randnarbe und durch deutliche konzentrische Ringe gekennzeichnet. Ihre mäßig scharfwinklige Basis hat eine ausgesprochen kleinflächig facettierte Schlagfläche. Außerdem zeichnet sich dieser regelmäßig rechteckige Abschlag mit relativ feiner dexrolateraler Schneide vor allem durch eine sinistrolaterale steil retuschierte Kerbe und durch ein partiell retuschiertes terminales Ende aus (L. 41, B. 45, D. 9). In der Nähe der erwähnten Feuerstätte wurde auch eine Klinge mit abgebrochener Spitze gef unden, die merkwürdigerweise bereits aus Feuerstein hergestellt ist. Sie ist durch eine tiefe dextrolateral-terminale steil retuschierte Kerbe, sowie durch eine weitere dextrolateral-basale, ventral gerichtete seichte Kerbe gekennzeichnet. Die Gesamtprägung der Bearbeitungstechnik dieser besprochenen Artefakte zeugt für eine relativ entwickelte Form des Le vallo is ie ns. Es liegen hier somit die ersten stratigraphisch erfaßten Funde dieser Kultur nicht nur im Karpatenbecken, sondern auch in der Tschechoslowakei vor.

Bei der erwähnten Rettungsforschung, die im Winter bei der Lehmgewinnung am Profil O7 erfolgte, fand man auch spärliche Stïcke, die sowohl typologisch als auch stratigraphisch in den Moustie $\mathrm{r}$ - Kreis eingeordnet werden können. Dabei muß man in Betracht ziehen, daß sie aus PK III + II vom Grenzabschnitt der Schichten B 1/2 stammen. 


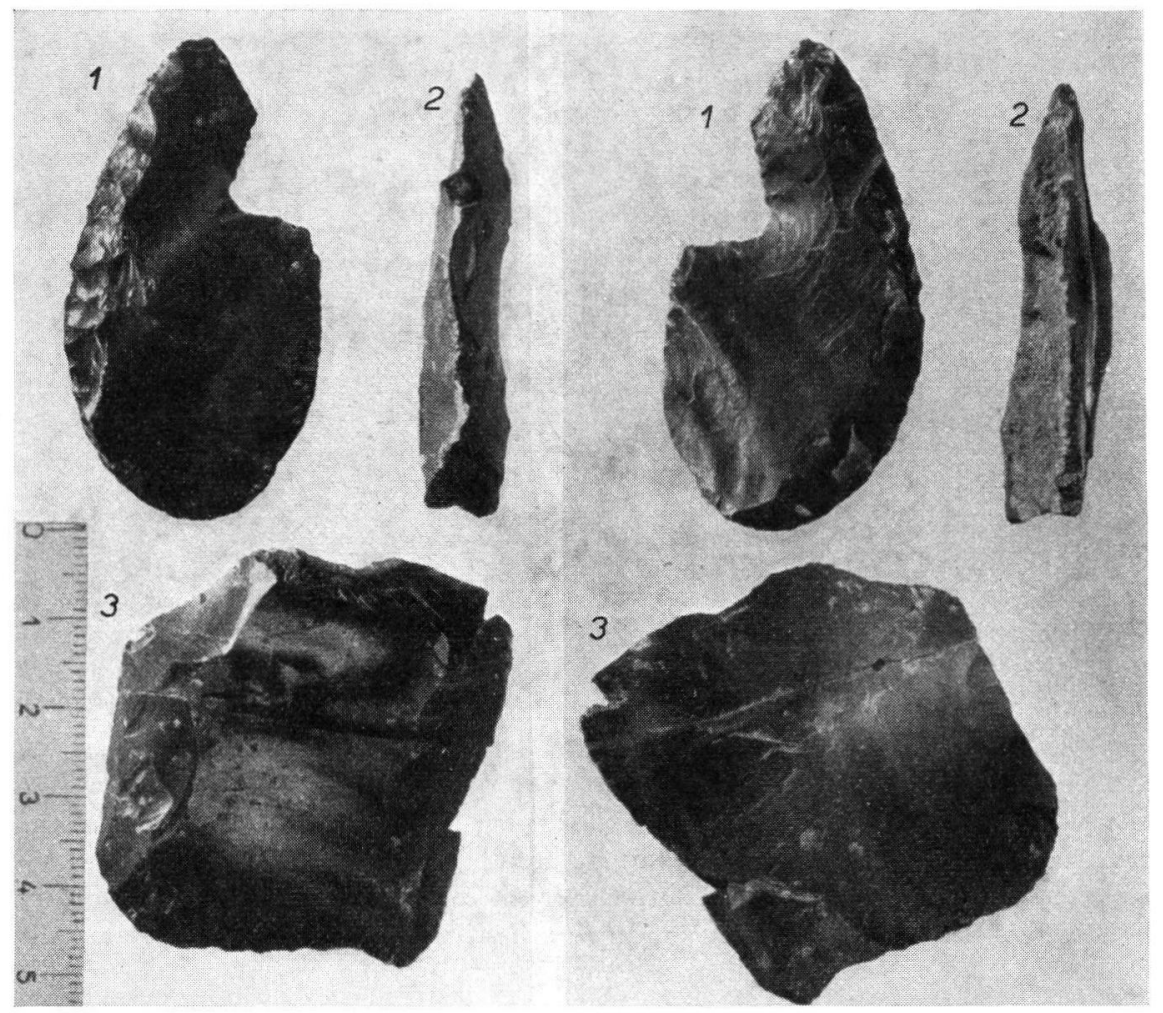

Abb. 6. 1 Szeleta-Blattspitze aus der Schicht B2-k (Profil 03); 2 Gravette-Spitze, die unter dem Profil 07 gefunden wurde; 3 Mit Levallois-Technik bearbeitetes Artefakt aus der Schicht C2-b (Profil 07).

Foto З̌оснн.

Typologisch liegt hier eine Moustie r-Spitze mit auffallend hohem Rücken vor; von der rechten Seite erinnert diese durch Negative nach breiten kurzen Klingen an ein Kernstück, welches durch seine nach außen bogenförmig gewölbte rechte Seite auch die Funktion eines Moustier-Schabers ausüben kann. Das terminale Ende der Spitze ist mäßig retuschiert und die Angehörigkeit zur moustéroiden Gruppe wird auch durch die vertikale stichelartige, sekundär retuschierte Fläche an der linken Kante hervorgehoben. Die einseitige stichelartige Fläche der Moustie r-Spitzen ist auch an anderen slowakischen Moustier-Fundstellen, wie in Komjatice oder namentlich im stratigraphisch erfaßten Bojnice bekannt (J. BÁRTA, 1961). Die angeführte unifaziale Spitze (Abb. 7/1) besteht aus grüngrauem Radiolarit (L. 80, B. 47, D. 34). Ein weiterer Fund aus dieser Lage ist ein größerer, diskoid abgeschlagener Geröllsegmentabschlag mit Negativen nach diskoiden Abschlägen an der Dorsalseite. Ventral hat dieser Abschlag einen auffallend großen Doppelbulbus mit mehreren konzentrischen Schlagsprüngen und großer gedoppelter Schlagnarbe, sowie mehrfachen konzentrischen Ringen. Dorsal blieb noch zum Teil die ursprüngliche Verwitterungsrinde des Radiolaritgerölles erhalten, während die Ventralseite gelbgrün gefärbt und gelblich patiniert erscheint (L. 68, B. 61, D. 20).

Der stratigraphische Wert des Profils von Nové Mesto wird auch durch Funde von S z el e ta-Artefakten erhöht, welche von der Oberfläche von PK III + II, wahrscheinlich aus der Schicht B2-i stammen. An der Oberfläche von PK II wurden Anhäufungen von Holzkohlen und vereinzelte, bisher typologisch unbestimmbare Radiolarit-Abschläge festgestellt. KošNÁR's Fund einer Blattspitze im Herbst 1960, der aus sekundärer Lage 

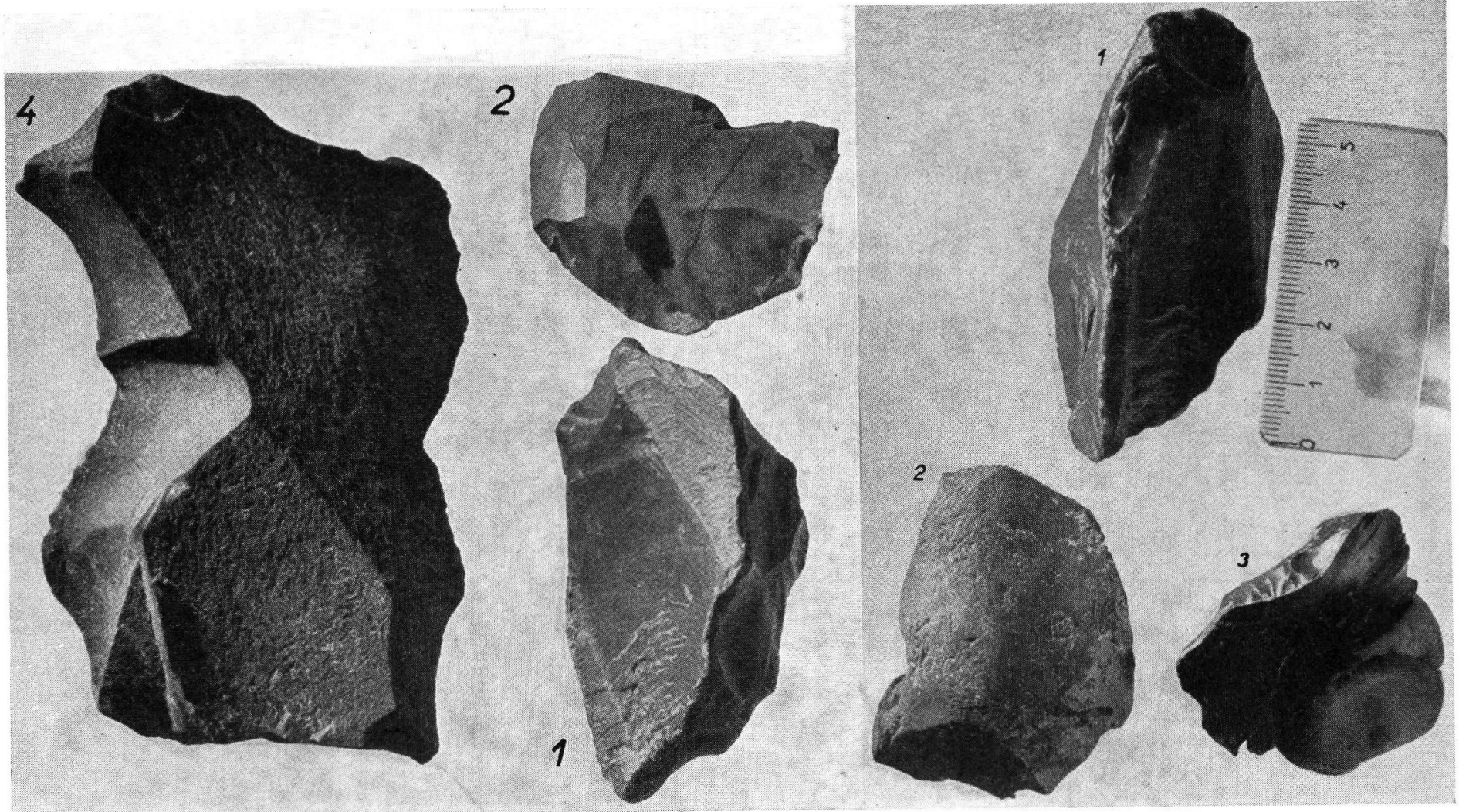

Abb. 7.1 Moustier-Schaber aus der Schicht B2-f (Profil 07); 2 Gravette-Kernstïck aus Radiolarit (Schicht B3, Profil 07); 3 Mit Levallois-Technik bearbeitetes Artefakt aus der Schicht c2-b(Profil 07); 4 Clactonartiger Abschlag aus der Schicht C1-c (Profil 01).

Foto క̌косн. 
stammt, beweist verläßlich die Anwesenheit von Sz e le ti e n an der untersuchten Fundstelle. Anhand der Sedimentreste, die an der Blattspitze hafteten, konnte festgestellt werden, daß dieser Fund aus den Lehmbröckelsanden im oberen Abschnitt von PK II stammt. Die Szeleta-Blattspitze von Nové Mesto (Abb. 6a) weist eine mäßige Krümmuıı auf, die für die Entstehung aus einer breiten flachen Klinge von rotbraunem Radiolarit zeugt. Sie hat eine regelmäßig ovaloide Basis, welche ventral und dorsal partiell beatbeitet ist und in eine symmetrische Spitze übergeht, die vor allem ventral eine stärker uuppenretusche aufweist. Die ursprüngliche Klinge wurde nach dem Zuspitzen an den Rändern nur partiell durch eine ziemlich regelmäßige Flächenretusche bearbeitet. Eine dextrolateralterminale, wahrscheinlich sekundäre Kerbe wurde insbesondere ventral sekundär leicht flächig retuschiert (L. 54, B. 29, D. 5). Angesichts ihrer ovaloiden Basis hat die Blattspitze von Nové Mesto bisher keine Analogie im slowakischen Raum. Sie steht den ungarischen von Nové Mesto bisher keine Analogie im slowakischen Raum. Sie steht den ungarischen Funden von der Jankovich-Höhle (M. GábоRI, 1954), eventuell den mährischen von Ondratice nahe, wo einige Analogien bekannt wurden (ČERvinKA, 1927). Mit den erwähnten Anhäufungen von Holzkohlen stehen offenbar auch Lagen von gebranntem Lehm vom Jahr 1944 in Zusammenhang, auf die der Geograph M. Lukniš mündlich hingewiesen hat.

Die zahlreichsten archäologischen Funde an der Lokalität von Nové Mesto stammen aus dem schwach ausgeprägten Bodenkomplex PK I aus den Schichten B3-bc, in denen auch vereinzelt Holzkohlen festgestellt wurden. Die Industrie aus dieser Lage besteht aus häufigen Abschlägen und Kernstücken verschiedener Typen, die größtenteils aus gelben, grauen und grünlichen Radiolariten der weißkarpatischen Klippzone hergestellt wurden. Für den Gravette-Charakter zeugt außer den nichtretuschierten Klingen vor allem ein Stichelabschlag mit bogenförmig nach außen gewölbter Seite. Dadurch wird die Herstellung von Sticheln aus den Gravette-Kratzern gekennzeichnet (ABsolon, 1945). Analogien dieses Artefaktes sind von mehreren Grave t te-Rastplätzen in der Westslowakei und vor allem in Mähren bekannt. Die Anwesenheit von flachen Quarzitgeröllen mit schwachen Klopfspuren unterstiitzt den Beweis der Herstellung von G ravetteArtefakten an Ort und Stelle. Das Auftreten des Gravettiens von Nové Mesto im Schichtverband des Paudorfer Interstadials (PK I) bietet gleichzeitig einen Schlüssel zur Lösung der stratigraphischen Stellung von früher nicht erkannten (Zamarovce) und Oberflächenstationen des Gravettiens im Vorgebirge der Weißen Karpaten. Hier, d. h. im Abschnitt zwischen Púchov und Nové Mesto, kann das Benützen von lokalem Rohstoff Juraklippen-Radiolarit - an jungpaläolithischen Siedlungen häufig beobachtet werden. Die stratigraphisch erfaßten Funde von älteren Kulturen in Nové Mesto beweisen im besprochenen Gebiet eine lange Tradition im Benützen dieses einheimischen slowakischen Rohstoffes, welcher auch in beträchtlichem Umfang nach dem benachbarten Mähren exportiert wurde; allerdings sind die mährischen Funde stratigraphisch bisher nicht erfaßt (SKUTIL, 1947).

Die angeführte Übersicht über die archäologischen Funde im Lößprofil von Nové Mesto nad Váhom stellt nur die erste Phase der Forschungen dar, die mit Rücksicht auf die außerordentliche Bedeutung dieser Fundstelle in der Zukunft fortgesetzt werden müssen. Mehrere paläolithische Horizonte und ihre Verknüpf ung mit ausgeprägten fossilen Bodenkomplexen verleihen dem Profil von Nové Mesto eine Schlüsselstellung unter den bisher bekannten Lößprofilen des Karpatenbeckens. Vor allem muß die feste stratigraphische Einstufung von Altpaläolithikum hervorgehoben werden, die hier zum erstenmal in diesem Raum durchgeführt wurde.

\section{Stratigraphische Auswertung des Profils}

Die stratigraphische Auswertung des Profils stützt sich auf folgende Kriterien:

1) Analogien in der Ausbildung der Bodenkomplexemit klassischen Fundstellen in Böhmen und Mähren 
2) Zweidurch Molluskenfunde belegte Interglazialhorizonte verschiedenen Alters.

Auf Grund der angeführten Kriterien gehört die jüngere interglaziale Fauna sicher dem letzten Interglazial (Eem-I.) an, da sie an der Basis einer charakteristisch ausgebildeten Abfolge von Bodenkomplexen PK II und PK III auftritt. Die ältere warmzeitliche Fauna ist vorletztinterglazial, da sie von PK IV mit charakteristischen Hauptmerkmalen überlagert wird.

Eine nähere Einstufung erfolgte im Sinne des zyklischen stratigraphischen Systems der Lößserien, das genauer in einem selbständigen Aufsatz besprochen wird (KUKLA, 1961a). Dieses System stützt sich auf die Beobachtung, daß die Abfolge der Sedimentations- und Bodenbildungsvorgänge während des Quartärs eine überraschend detaillierte Wiederholung in gesetzmäßigem Zyklus aufweist. Die Zyklen B 1, B 2 und B 3 sind mit Soergei.s theoretischer Auffassung der Perioden R/W - W 1, W 1/2 - W 2 und W 2/3 - W 3 annähernd identisch. Der ältere Zeitabschnitt $M / R$ - $R$ zerfällt aber in zwei Interglaziale und folgende Glaziale, wobei jedes der beiden Paare in der zyklischen Auffassung der letzten Warmzeit + Kaltzeit gleichwertig ist. Es gibt hier deshalb sechs Zyklen zweiter Ordnung, die stets mit einem Boden der Lessivé-Entwicklungsreihe an der Basis beginnen. Die aufgeschlossene Basis unseres Profils reicht somit nicht tiefer als in den Löß D 3, der jünger als die Holstein-Warmzeit sein muß. Beide Lessivés von PK IV dürften denjenigen Böden entsprechen, die in Süddeutschland in die Altriß/Jungriß-Warmzeit eingeordnet werden und mit denen im nordischen Raum vielleicht die als Ohe-Interglazial aufgefaßten Bildungen identisch sind.

Die stratigraphische Eingliederung der einzelnen Schichten ergibt sich aus der Tafel 1. An dieser Stelle wird deshalb nur die Einstufung der archäologischen Fundhorizonte besprochen:

1) Der jüngste Horizont in PK I (B3-bc) liegt an der Oberfläche des parautochthonen Hauptbodens des Mittelwürm-Interstadials (=Paudorf, W 2/3), also in entsprechender Lage wie in Pollau, Unter-Wisternitz und anderswo. Die im Profil aufgefundenen Artefakte sind zwar typologisch nicht eindeutig, unter der Lößwand wurde jedoch eine typische Grave t t e-Klinge gefunden.

2) Der ältere Horizont in der Oberflächenschicht des autochthonen Humusbodens B2-i ist durch verschleppte linsenartige Holzkchlenschlieren und Klümpchen von gebranntem Lehm gekennzeichnet. In der nächsten Nachbarschaft dieses Horizontes, unweit des Abschuittes O3, wurde eine $\mathrm{Sz}$ ele ta-Spitze gefunden. Aus den petrographischen Merknıalen der abgekratzten Lehmreste ergibt sich eine Übereinstimmung mit den Proben O316 und O3-17, also mit der Schicht der Lehmbröckelsande. Die Holzkohlen sind auch im hangenden Marker und Lehmbröckelsand verstreut, eine größere Holzkohlenanhäufung, die als verschleppte Feuerstätte angesprochen werden dürfte, ist hier aber nicht vorhanden.

3) Der dritte Fundhorizont befindet sich an der Basis des gefleckten Humusbodens B2-ghi und in den lichter gefärbten Lehmen B 1 / 2-f. Die Artefakte wurden von keinem Kulturobjekt begleitet, das auf ihre primäre Lagerung deuten würde. Man kann also nicht ausschließen, daß beide Stücke aus einem ursprünglich tieferen stratigraphischen Niveau umgelagert wurden. Es handelt sich hierbei um M ous ti e r - Artefakte.

4) Im Lessivé B1-a wurden verstreute gröbere Holzkohlen in einer stratigraphischen Lage gefunden, deren Position den Fundschichten von Sedlec bei Prag und von Gánovce verwandt ist. Bisher wurde zwar kein Artefakt festgestellt, der Horizont muß aber angesichts des Menschenaufenthaltes weiter verfolgt werden.

5) Im Horizont C2-b wurden unstreitige Le vallo is-Geräte in der Nähe einer großen Holzkohlenakkumulation an einer Fläche etwa $1 \mathrm{~m}^{2}$ festgestellt, die von gebranntem Lehm begleitet wird. Es lagen offenbar Reste einer Feuerstätte vor. In demselben 
Horizont sind verstreute Holzkohlen und linsenartige Holzkohlenanhäufungen fast in gesamtem Raum der Ziegeleigrube vorhanden. Die Gleichzeitigkeit der Besiedlung mit der Schichtbildung ist hier also gesichert.

6) Die Holzkohlen in den Lagen C1-f und C1-g wurden bisher von keinen Artefakten begleitet und dürfen deshalb nur als Anzeichen der möglichen Menschenanwesenheit an der Fundstelle im betreffenden Zeitabschnitt betrachtet werden.

7) In der Pararendsina C1-c wurden Cla c t o n - Geräte entdeckt. Holzkohlen treten in dieser Schicht nur sehr vereinzelt auf.

\section{Schluß}

Die grundsätzliche Bedeutung des Profils von Nové Mesto für die Stratigraphie der Waagtallösse unterliegt keinem Zweifel. Es handelt sich um die erste slowakische Fundstelle, die ein paläontologisch belegtes vorletztes Interglazial $\mathrm{e} n \mathrm{th}$ ä $1 \mathrm{t}$, das hier überdies von gut ausgebildeten Ablagerungen eines paläontologisch belegten letzten Interglazials überlagert wird. Es liegt hier die einzige tschechoslowakische Lokalität vor, an der gut gegliederte Schichtpakete der frühen Abschnitte (Phase 1 des zyklischen Systems) des letzten und vorletzten Interglazials erhalten sind, in denen unter den Basis-Lessivés (Phase 2) Pararendsinen auftreten. Eine grobe Parallelisierung der Schichtenfolgen mit klassischen Profilen in Böhmen und Mähren ist durchaus verläßlich und die Identität der Bodenkomplexe PK II, PK III und PK IV mit entsprechenden Schichtpaketen der erwähnten Profile ist vollkommen klar. Aus diesem Grund geht somit hervor, daß hier das letzte und vorletzte Interglazial und nicht die älteren Warmzeiten vorliegen. Im Gegensatz zu böhmischen und mährischen Fundstellen sind hier die Humusböden auffallend schwach ausgebildet. Der Humushorizont kann stellenweise vollkommen fehlen. PK II ist schlecht in sich gegliedert und eine durchlaufende Lößschicht (=Löß W 1 in der Auffassung von ProšEk \& Ložek 1957) zwischen PK II und PK III ist nicht ausgebildet. Der parautochthone Boden der 5. Phase von B 1 ist deutlich ausgeprägt. Die Bodenkomplexe PK III und PK II fließen deshalb fast zusammen und konnten ohne eingehende petrographische Untersuchung bisher nicht verläßlich unterschieden werden.

Die Hauptbedeutung der Fundstellen ist allerdings in den Funden von menschlichen Kulturresten $\mathrm{zu}$ sehen, die bisher in fünf übereinander liegenden Horizonten festgestellt wurden. Die jüngste Industrie ist das Gravettien. Tiefer folgen das Szeletien, Moustérién und protolithische Industrien in PK IV, wovon die jüngere Levallo is - Merkmale aufweist, die ältere mit Cla cton-Technik bearbeitet ist. Alle Fundhorizonte können relativ genau mit einzelnen Schichten der Bodenkomplexe verknüpt und geologisch datiert werden. Von diesem Gesichtspunkt aus muß der Ziegeleigrube von Nové Mesto nad Váhom im mitteleuropäischen Raum eine Sonderstellung beigemessen werden.

\section{$\mathrm{S} c \mathrm{hriftenverzeichnis}$}

Absolon, K.: Výzkum diluviální stanice lovců mamutů v Dolních Věstonicích na Pavlovských Kopcích na Moravě, Pracovní zpráva za třetí rok 1926. - 252 S., Brno 1945.

Ambrož,V., LožEK, V. \& Prošek, Fr.: Pléistocène récent aux environs de Moravany près Piešt'any sur le Váh (Slovaquie occidentale). - Anthropozoikum 1, S. 53-142, Taf. I-IV, Praha 1952.

BÁrta, J.: Industria moustierskeho okruhu na západnom Slovensku. - Památky archeologické 52, S. 31-39, Praha 1961.

Ċervinka, J. L.: Pravěk zemí českých. - 80 S., Brno 1927.

Gábori, M.: Sol'utrejskaja kul'tura Vengrii. - Acta Archaeologica Acad. Scient. Hung. 3, 68 S. (Sonderdruck), Budapest 1954. 
KLíMA, B.: Übersicht über die jüngsten paläolithischen Forschungen in Mähren. - Quartär 9, S. 85130, Taf. IV-X, Bonn 1957.

KuKLA, J.: Quaternary Sedimentation Cycle. - Survey of Czechoslovak Quaternary, S. 145-154, Warszawa 1961 (1961a). - Lithologische Leithorizonte der tschechoslowakischen Lößprofile. - Věstník Ustředního ústavu geologického 36, 5, S. 369-372, Taf. I-II, Praha 1961 (1961b).

KuKLA, J. \& LožEK, V.: Loesses and Related Deposits. - Survey of Czechoslovak Quaternary, S. 1128, 5 Beil., Pl. I-III, Warszawa 1961.

KuKLA, J., LožEK, V. \& Ż́RUBA, Q.: Zur Stratigraphie der tschechoslowakischen Lösse. - Quartär $12, \ldots-\ldots$, Taf. ... - ..., Bonn 1961.

LožEK, V. \& KuKLA, J.: Das Lößprofil von Leitmeritz an der Elbe, Nordböhmen. - Eiszeitalter und Gegenwart 10, S. 81-104, 1 Taf., Öhringen 1959.

LožEK, V. \& TrRáčEK, J.: Př́íspěvek k poznání vývoje údolí Váhu mezi Trenčínem a Piešt'any (Contribution to the Knowledge of Development of the Valley of the River Váh between Trenčín and Piešt'any). - Sborník Československé společnosti zeměpisné 65,1 , S. 6-14, 2 Taf., Praha 1960.

Ložek, V., Trráčex, J. \& FEjFAr, O.: Die quartären Sedimente der Felsnische auf der Velká Kobylanka bei Hranice (Weißkirchen). - Anthropozoikum 8, S. 177-203, Taf. I-II, Praha 1959.

Luknıš, M.: Poznámky ku geomorfologii Beckovskej brány a pril'ahlých území (Quelques remarques sur la géomorphologie de la Porte de Beckov et des régions avoisinantes). - Práce Śtátneho geologického ústavu 15, 32 S., 5 Taf., 1 Karte, Bratislava 1946.

Pelíšex, J.; Spraše dolního Pováží (The Loess in the River Valley of Lower Váh in Czechoslovakia). - Geologický Sborník SAV 3, S. 87-99, Taf. XV-XIX, Bratislava 1953.

Petriok, J,: Měkkýši slovenského kvarteru/Sdělení první /. - Rozpravy II. tř́ídy České akademie 44, 15, 16 S., Taf. 1, Praha 1935. - - Kvarterní měkkýšsi Trenčína, Trenčianských Teplic a okolí. - Ebendort, 45, 13, 14 S., Praha 1936.

Prošex, F.: Nové paleolitické stanice na východním Slovensku. - Archeologické rozhledy 5, S. 289-297, Praha 1953 (1953a). - - Le Szeletien en Slovaquie. - Slovenská Archeologia 1, S. 133-194, Bratislava 1953 (1953b).

Prošex, F. \& Ložek, V.: Sprašový profil v Bance u Piešt’an (západní Slovensko) (The Loess Section at Banka near Piešt'any [Western Slovakia]). - Anthropozoikum 3, S. 301-323, Taf. I-II, Praha 1954. - - Výzkum sprašového profilu v Zamarovcích u Trenčína (Untersuchung des Lößprofiles von Zamarovce bei Trenčín). - Anthropozoikum 4, S. 181211, Taf. I-II, Praha 1955. - - Stratigraphische Ubersicht des tschechoslowakischen Quartärs. - Eiszeitalter und Gegenwart 8, S. 37-90, OOhringen 1957.

SkutıL, J.: Karpatské rádiolaritové vlárske paleolitikum moravské. - Historica Slovaca 5, S. 16-33, Bratislava 1947.

Woldstedt, P.: Das Eiszeitalter, II. Bd. - 2. Auflage, 438 S., 1 Taf., Stuttgart 1958.

ZotZ, L. \& VLK, W.: Das Paläolithikum des unteren Waagtales. - Quartär 2, S. 65-101, Taf. XVXIX, Berlin 1939.

ŽEBerA, K.: Nejstarší památky lidské práce z Čech. - Rozpravy Ústředního ústavu geologického 14, 84 S., 42 Taf., 1 Karte, Praha 1952.

Manuskr. eingeg. 29. 6. 1961

Anschrift der Verfasser:

Dr. Jiř́ Kukla, Archäologisches Institut der ČSAV, Prag 1, Letenská 4.

Dr. Vojen Ložek, Geologische Zentralanstalt, Prag 1, Malostranské náměstí 19.

Dr. Juraj Bárta, Archäologisches Institut der SAV, Nitra, Hrad (Slowakei) Tschechoslowakei. 